\title{
Pultrusion process of natural fibre-reinforced polymer composites
}

\begin{abstract}
In this chapter a review of pultrusion process is presented. The process can be used to produce composites with reinforcements that are either synthetic fibres such as glass and carbon fibres or natural fibres like kenaf, jute, and hemp fibres. Selected previous work such as mechanical properties, processing parameters, and product development of pultruded natural fibre composites is reviewed. Special attention is paid on the development and properties of kenaf fibre composites fabricated from the pultrusion process
\end{abstract}

Keyword: Pultrusion; Natural fibre composites; Kenaf; Biopolymers; Product development 